\title{
PERSONALIDAD Y SU RELACIÓN CON EL USO VERSUS ABUSO DEL TELÉFONO MÓVIL
}

\section{RELATION PERSONALITY TO MOBILE PHONE USE AND ABUSE}

\author{
Ma Angustias OLIVENCIA-CARRIÓN $^{1}$, \\ $M^{a}$ Nieves PÉREZ-MARFIL ${ }^{1}, M^{a}$ BelÉn RAMOS-REVELLES ${ }^{1} Y$ \\ FRANCISCA LÓPEZ-TORRECILLAS ${ }^{1}$
}

Cómo referenciar este artículo/How to reference this article:

Olivencia-Carrión, M. A., Pérez-Marfil, M. N., Ramos-Revelles, M. B., López-Torrecillas, F. (2016). Personalidad y su relación con el uso versus abuso del teléfono móvil [Relation personality to mobile phone use and abuse]. Acción Psicológica, 13(1), 109-118. http://dx.doi.org/10.5944/ap.13.1.17427

\section{Resumen}

Las investigaciones realizadas han confirmado que el uso excesivo del teléfono móvil genera efectos perjudiciales en la salud y en las relaciones sociales. El objetivo del presente estudio es analizar las variables de personalidad asociadas al uso versus abuso del teléfono móvil. Ciento sesenta y cinco participantes fueron divididos en tres grupos en función del tiempo de uso diario del teléfono móvil, el Grupo 1 usaba el teléfono móvil menos de una hora al día, el Grupo 2 usaba el teléfono móvil menos de dos horas al día, y el Grupo 3 usaba el teléfono móvil más de cuatro horas al día. Los resultados mostraron que el Grupo 3 presentaba puntuaciones bajas en Estabilidad, Atención a las Normas, Perfeccionismo y Autocontrol y

puntuaciones altas en Animación y Extraversión y el Grupo 2 presentaba puntuaciones altas en Extraversión y Ansiedad. Se resalta la implicación de la personalidad en la adicción al móvil.

Palabras clave: adicción; teléfono móvil; personalidad; tecnologías de información y comunicación (TIC).

Abstract
Research has confirmed that excessive use of these
devices can cause harmful effects on health and social
relations of users. The aim of this study is to analyze
the personality variables associated with the use
versus abuse of mobile-phone. The sample consisted

Correspondencia Francisca López-Torrecillas. Departamento de Personalidad, Evaluación y Tratamiento Psicológico. Facultad de Psicología. Campus Universitario de la Cartuja s/n. Universidad de Granada.

Email: fcalopez@ugr.es

${ }^{1}$ Universidad de Granada, España.

Recibido: 20 de octubre de 2015.

Aceptado: 20 de diciembre de 2015 . 
of a total of 165 participants that were divided into three groups depending on the time of daily mobile phone use, the Group 1 using the mobile phone less than an hour a day, the Group 2 using the mobile phone less than two hours a day, and Group 3 using the mobile phone more than four hours a day. The results showed that Group 3 had low scores on Stability, Attention to the Rules, Perfectionism and Selfcontrol and high scores in Animation and Extraversion and Group 2 had higher scores on Extraversion and Anxiety. This study demonstrated the involvement of personality in addiction mobile-phone.

Keywords: addiction; mobile-phone; personality; information and communication technologies (ITC).

\section{Introducción}

El teléfono móvil se ha convertido en un componente más de los elementos esenciales que forman parte de la vida cotidiana e íntima (comunicación, juego, fotografía, participación en redes sociales, descarga y escucha de música, visualización de películas y telefilmes, apuestas online, radio, noticias, etc.). Pocos elementos a lo largo de la historia han resultado ser tan importantes en el día a día del ser humano, pueden ser determinantes en la identidad del individuo y tan esenciales, que pueden crear relaciones de dependencia y apego emocional. Además, pueden estar provocando numerosos casos de dependencia en personas que obtienen, de estas herramientas, un refugio que las aleja de sus problemas emocionales o familiares. Se trata de conductas repetitivas que resultan placenteras en las primeras fases, pero que después no pueden ser controladas por el individuo. Nunca antes un aparato tecnológico se había convertido en un aspecto tan importante en la vida diaria de las personas (Chóliz, 2012; Srivastava, 2005).

El uso problemático del teléfono móvil y sus repercusiones psicológicas ha sido un foco de interés en la literatura científica, donde los criterios diagnósticos para las adicciones comportamentales se han discutido ampliamente. En el caso del uso de los teléfonos móviles, las razones que han llevado a considerarlo como una conducta adictiva se refieren principalmente al tiempo y a la atención empleada por los usuarios en sustitución de otras conductas o actividades, con la consiguiente pérdida de control (Bononato, 2005; Echeburúa, Salaberría y Cruz-Sáez, 2014; Ruiz-Olivares, Lucena, Pino y Herruzo, 2010; Sánchez-Carbonell, Beranuy, Castellana, Chamarro y Oberst, 2008; Srivastava, 2005; Viñas y González, 2010). Labrador y Villadangos (2010) llevaron a cabo un estudio con el objetivo de evaluar la percepción subjetiva de peligro derivada del uso de las Nuevas Tecnologías (NT) en 1710 adolescentes de 12 a 17 años de la Comunidad de Madrid. Para evaluar la adicción a las NT se utilizó el Cuestionario de Detección de Nuevas Adicciones (DENA), diseñado para este estudio. Se encontró una correlación positiva entre el tiempo de uso y la percepción del problema y que la edad correlacionaba positivamente con esta percepción. Se constató que las NT generan conductas similares a las consideradas características en las adicciones (como por ejemplo el uso del teléfono móvil). Los autores evidencian la relajación producida por el uso del teléfono móvil y/ o el malestar si no puede utilizarse. Observaron también que los usuarios que abusan del teléfono móvil están de manera constante casi obsesiva comprobando el teléfono. Los adolescentes son los usuarios más vulnerables puesto que aún no tienen un control completo de sus impulsos, son más fácilmente influenciables por campañas publicitarias y comerciales y han aceptado el teléfono móvil como un símbolo de estatus, provocando sentimientos negativos y problemas de autoestima cuando no tienen teléfono móvil o que no reciben tantos sms o llamadas como sus compañeros (Muñoz-Rivas y Agustín, 2005). Diversos estudios (Bianchi y Phillips, 2005; Chóliz, 2012; Hong, Chiu y Huang, 2012; Ishfaq, Tehmina y Khadija, 2011; Jenaro, Flores, Gómez-Vela, González-Gil y Caballo, 2007; Leung, 2007; Turner, Love y Howell, 2008) han puesto de manifiesto que ciertas características de personalidad aumentan la vulnerabilidad psicológica para el desarrollo de una conducta adictiva. Específicamente, Bianchi y Phillips (2005) llevaron a cabo un estudio con 194 participantes (132 mujeres y 62 varones), con edades entre 18 y 85 años, cuyo objetivo fue relacionar el uso versus abuso del teléfono móvil con la personalidad. Para evaluar dicha relación se utilizó la Escala de Potencial de 
Adicción. (APS; Weed, Butcher, McKenna, 1992), el Cuestionario de Autoestima de Coopersmith (1989), el Cuestionario de Personalidad de Eysenck (EPQ; H. J. Eysenck y S. B. G. Eysenck, 1991) y la Escala de Uso Problemático del Móvil (MPPUS; Bianchi y Phillips, 2005), desarrollada para esta investigación. Los resultados mostraron que la extraversión y la autoestima explicaban tanto el uso general, como el uso problemático (evaluado por el tiempo que el usuario dedica al uso del teléfono móvil). Puntuaciones altas en neuroticismo y bajas en autoestima están relacionadas con el uso problemático del teléfono móvil. Inesperadamente, el neuroticismo no se relacionó con el tiempo autoinformado dedicado al uso del teléfono móvil, ni con el uso problemático. En la misma línea, Leung (2007) analizó las variables de personalidad para predecir el comportamiento adictivo en el uso del teléfono móvil. Los participantes fueron 624 jóvenes con edades comprendidas entre 14 y 28 años que respondieron a una entrevista telefónica de 27 ítems desarrollada a partir de dos escalas de Adicción al Móvil, una de Bianchi y Phillips (2005) y la otra de Young (1996). Se encontró que la adicción al teléfono móvil estaba inversamente relacionada con la autoestima y directamente relacionados con la búsqueda de sensaciones y el aburrimiento. Se señala también que las características más comunes en los patrones de uso del teléfono móvil son los mensajes de texto, el entretenimiento y la búsqueda de información. El estudio de Jenaro et al. (2007) evalúa el abuso de Internet y del teléfono móvil en una muestra de 337 estudiantes universitarios. En el estudio se desarrollan dos medidas, la primera es la Escala de Abuso de Internet (IOS) y la segunda es la Escala de Abuso del teléfono móvil (CDS). Otras medidas utilizadas fueron el Inventario de Ansiedad de Beck (Beck y Steer, 1993a), el Inventario de Depresión de Beck (Beck y Steer, 1993b) y el Cuestionario General de Salud-28 de Lobo, Pérez-Echeverría y Artal (1986). Hallaron que el abuso de Internet estaba asociado con altos niveles de ansiedad y que el abuso del teléfono móvil estaba asociado con altos niveles de ansiedad e insomnio; encontraron también diferencias de sexo, las mujeres abusaban más del teléfono móvil que los hombres. Siguiendo en la misma dirección, Turner et al. (2008) realizaron un estudio cuyo objetivo fue analizar cómo las características de personalidad de los individuos que se relacionan con el uso versus abuso de la tele- fonía móvil. La muestra incluyó a 184 estudiantes universitarios (58 hombres y 126 mujeres) con una edad entre 17 y 43 años. Se utilizó la Escala de Afecto Positivo y Negativo (PANAS; Watson, Clark, Tellegen, 1988), el Cuestionario de Personalidad de Eysenck (EPQ-R; Francis, Brown, Philipchalk, 1992) y el Cuestionario de Actitudes Generales del uso de Teléfono Móvil (Howell, Love y Turner, 2005). Se encontró una relación entre psicoticismo e inhibición reducida sobre el uso del teléfono móvil en los contextos sociales y que el neuroticismo y la extraversión influían en el abuso del teléfono móvil; la extraversión se relacionó con una mayor frecuencia de uso del teléfono móvil pero no con habilidades como facilidad de uso social o de confort al realizar y recibir llamadas en lugares públicos. En el estudio de Ishfaq et al. (2011) se analizaron los patrones de comportamiento adictivos de uso del teléfono móvil (a través de una entrevista diseñada para este estudio) en una muestra de 400 estudiantes (38.2\% hombres) de edades comprendidas entre 18 y 26 años. Encontraron que el $62.7 \%$ de la muestra analizada fracasaba en sus esfuerzos por reducir el uso del teléfono móvil, el $27.8 \%$ preferían el contacto del teléfono móvil y evitaban el contacto cara a cara con sus amigos, el $36.8 \%$ afirmaron que los demás se quejaban acerca de su uso excesivo, el 31.1 \% sufría una disminución en su productividad debido al uso del teléfono móvil. Concluyeron que la mayoría de los jóvenes utilizaban el teléfono móvil como estrategia de afrontamiento de estados emocionales negativos tales como aburrimiento o disforia. El estudio de Chóliz (2012) tuvo como objetivo analizar las características de personalidad que aumentan la vulnerabilidad para el desarrollo de adicciones psicológicas; para ello desarrolló un cuestionario basándose en los criterios de dependencia de los trastornos adictivos del DSM-IV-TR (APA, 2011) cuyo fin fue diagnosticar la adicción al teléfono móvil en función de la abstinencia, falta de control, tolerancia e interferencia con otras actividades. Encontraron una relación directa entre el grado de dependencia, bajas puntuaciones de control de los impulsos y de autoestima y altas puntuaciones de búsqueda de sensaciones. Además el teléfono móvil se usaba como estrategia de afrontamiento para evitar los estados de ánimo desagradables. Hong et al. (2012) con una muestra de 269 estudiantes universitarias (mujeres) analizaron la relación entre la personalidad y el uso adictivo del teléfono móvil. 
Utilizaron la Escala de Medición para el Comportamiento de Uso del Teléfono Móvil (Walsh, White, Cox y Young, 2011), la Escala de Adicción al Teléfono Móvil (MPAS; Young, 1998), la Escala de Autoestima de Rosenberg (Rosenberg, 1965) y Escala de Personalidad de Lai y Lai (2003). Los resultados indicaron que la extraversión y la ansiedad predicen, de manera positiva, la adicción al teléfono móvil y que la autoestima predice, en sentido inverso, la adicción al teléfono móvil. Otras variables tales como los problemas para el manejo del tiempo y las dificultades académicas están relacionadas con dicho comportamiento adictivo.

En consonancia con lo anterior, el objetivo de este estudio fue examinar los patrones del uso y/o abuso de comportamientos susceptibles o indicadores de una adicción como el uso del teléfono móvil en función de la personalidad de los estudiantes universitarios de Granada.

\section{Método}

\section{Participantes}

La muestra estuvo compuesta por un total de 165 estudiantes de Psicología de la Universidad de Granada, con edades comprendidas entre los 18 y los 21 años (media 21.6 y desviación típica 2.96 . De ellos, $16 \%$ eran hombres y $84 \%$ eran mujeres. Los participantes fueron divididos en tres grupos en función del uso diario del teléfono móvil (de acuerdo con el cuestionario de Historia de Uso del Tiempo Libre). El Grupo $1(n=83)$ compuesto por los participantes que usaban el teléfono móvil menos de media hora; el Grupo $2(n=45)$ compuesto por los participantes que usaban el teléfono móvil como má- ximo dos horas al día y el Grupo $3(n=37)$ compuesto por los participantes que usaban el teléfono móvil más de cuatro horas al día. En la Tabla 1 pueden verse las características socio-demográficas de los grupos.

\section{Instrumentos de evaluación}

Historia del Uso del Tiempo Libre HUTL (diseñado para este estudio). Es un cuestionario diseñado para explorar el uso del tiempo libre, así como los posibles problemas asociados a las adicciones psicológicas, en jóvenes. Está formado por dos partes diferenciadas. La primera hace referencia a las actividades realizadas en el tiempo libre (Teléfono móvil, Internet, Ir de Compras, Hacer ejercicio fisico, Leer, Alquilar vídeos para verlos en casa, Jugar a la video consola, Viajar, Ir a conciertos, Ir al teatro, Escribir, Pasear con los amigos) y el sujeto tiene que señalar su frecuencia en la actividad en una escala tipo Likert de 0 a $7(0=$ Nunca la he realizado; $1=$ Sólo la realizó varias veces al año; $2=$ Varias veces al mes; $3=$ Varias veces a la semana; $4=$ Solo los fines de semana; $5=$ Diariamente pero no sabe cuánto tiempo; 6 = Máximo dos horas al día; 7 = Más de cuatro horas al día). La segunda parte del cuestionario evalúa las consecuencias psicológicas, sociales y familiares del abuso del teléfono móvil. Está formada por 13 ítems con 4 ó 7 alternativas de respuesta en los que el sujeto ha de elegir la opción que más le describe.

Cuestionario Factorial de Personalidad (16-PF-5; R. B. Cattell, A. K. S. Cattell y H. E. P. Cattell, 2005). Consta de 185 ítems con tres alternativas de respuesta (dos extremos y una alternativa intermedia señalada siempre con un interrogante"? Evalúa un espectro amplio de personalidad para mayores de 16 años y que tiene

Tabla 1

Características Sociodemográficas

\begin{tabular}{cccc}
\hline & & GRUPOS & G3 (> 4 horas) \\
\cline { 2 - 4 } & G1 (1/2 hora) & G2 (<2 horas) & Media (DT) \\
\hline Media (DT) & $22.49(2.5)$ & $20.48(2.91)$ & $20.91(3.36)$ \\
Sexo & $\mathrm{n}$ & $\mathrm{n}$ & $\mathrm{n}$ \\
Hombre & 23 & 43 & 1 \\
Mujer & 60 & & 36 \\
\hline
\end{tabular}


como finalidad la apreciación de dieciséis rasgos de primer orden (o escalas primarias de personalidad) y cinco dimensiones globales de personalidad (antes factores de segundo orden). Los dieciséis rasgos primarios son Afabilidad, Razonamiento, Estabilidad, Dominancia, Animación, Atención a las normas, Atrevimiento, Sensibilidad, Vigilancia, Abstracción, Privacidad, Aprensión, Apertura al cambio, Autosuficiencia, Perfeccionismo y Tensión. Las cinco dimensiones globales de personalidad son Extraversión, Ansiedad, Dureza, Independencia y Autocontrol. La comprensión de los constructos se facilita mediante una descripción a través de adjetivos de los polos o decatipos altos (+) o bajos (-). Incluye además tres medidas para evaluar estilos de respuesta para controlar los sesgos en las respuestas Manipulación de la imagen, Infrecuencia y Aquiescencia. La aplicación puede ser individual o colectiva con una duración de entre 40 y 45 minutos. De acuerdo con Aluja y Blanch (2003), esta quinta edición del 16-PF presenta buenas propiedades psicométricas, ha mejorado sensiblemente su validez y fiabilidad respecto a versiones anteriores. Se encuentra publicado en TEA Ediciones.

\section{Procedimiento}

La administración de los cuestionarios se llevó a cabo de forma colectiva, en grupos de 65-70 estudiantes de Psicología de la Personalidad ( $1^{\text {er }}$ curso) en la Facultad de Psicología (Universidad de Granada), durante el horario de prácticas en un tiempo de una hora. El estudio fue presentado a los participantes como una investigación sobre las diversas características de la personalidad y uso del tiempo libre, asegurándoles la confidencialidad de sus respuestas, así como el carácter voluntario de su participación. Los participantes proporcionaron consentimiento informado y la administración de las pruebas se realizó bajo la supervisión de una investigadora que aclaraba las dudas y enfatizaba la importancia de no dejar ninguna pregunta sin contestar. El estudio fue aprobado por el Comité de Ética de la Universidad de Granada. Los análisis estadísticos de este estudio han sido realizados a través del paquete estadístico SPSS en su versión 20 en castellano.

\section{Resultados}

Para comprobar las hipótesis, se realizó un Análisis Multivariado de la Varianza (MANOVA), para un diseño factorial, utilizando las variables Grupo [G1 (uso diario menos de media hora), G2 (uso máximo dos horas diarias), G3 (uso más de cuatro horas diarias)] como variable independiente y las variables derivadas del Cuestionario Factorial de Personalidad de Cattell (16-PF-5) como variables dependientes. Los resultados indicaron que había diferencias significativas entre los grupos (Wilks' Lambda $\left.=0.000, \mathrm{~F}_{24,139}=12804.338, p=.000\right)$ en las variables de personalidad. El análisis multivariado de la varianza para cada variable dependiente, mostró que había diferencias estadísticamente significativas en Estabilidad $\left(\mathrm{F}_{2,162}=3.411, \quad\right.$ Mce $\left.=1.744, \quad p=.035\right) ; \quad$ Animación $\left(\mathrm{F}_{2,162}=7.779\right.$, Mce $\left.=3.213, p=.001\right)$; Atención a las Normas $\left(\mathrm{F}_{2,162}=3.362\right.$, Mce $\left.=2.171, p=.037\right)$; Sensibilidad $\left(\mathrm{F}_{2,162}=3.268, \quad \mathrm{Mce}=.369, \quad p=.041\right), \quad \mathrm{Au}-$ tosuficiencia $\left(\mathrm{F}_{2,162}=3.568\right.$, Mce $\left.=1.848, p=.030\right)$; Perfeccionismo $\left(\mathrm{F}_{2,162}=6.495\right.$, Mce $\left.=2.213, p=.002\right)$; Tensión $\left(\mathrm{F}_{2,162}=3.963\right.$, Mce $\left.=1.663, p=.021\right)$; Infrecuencia $\left(\mathrm{F}_{2,162}=6.921, \quad\right.$ Mce $\left.=2.873, \quad p=.001\right) ; \quad$ Extraversión $\left(\mathrm{F}_{2,162}=7.173, \quad\right.$ Mce $\left.=2.612, \quad p=.001\right) ; \quad$ Ansiedad $\left(\mathrm{F}_{2,162}=6.154\right.$, Mce $\left.=3.614, p=.003\right) \quad \mathrm{y}$ Autocontrol $\left(\mathrm{F}_{2,162}=8.322, \quad \mathrm{Mce}=3.452, \quad p=.000\right)$. No se encontraron diferencias estadísticamente significativas en las variables Afabilidad, Razonamiento, Dominancia, Atrevimiento, Vigilancia, Abstracción, Privacidad, Aprensión, Apertura al Cambio, Aquiesciencia, Dureza e Independencia. En la Tabla 2 se puede observar las medias, desviaciones típicas, significación.

Los resultados de las pruebas de contraste "post hoc" de comparaciones múltiples entre los tres grupos (Tukey) pueden verse en la Tabla 2, donde se presentan los datos de comparación de los participantes para cada de personalidad que resulto ser significativa. Encontramos que el Grupo 3 presentaba las puntuaciones más bajas en Estabilidad en comparación con el Grupo 1. En Animación encontramos que el Grupo 3 presentaba puntuaciones más altas que el Grupo 1, a su vez, el Grupo 2 presentaba también las puntuaciones más altas que el Grupo 1. En Atención a las Normas el Grupo 3 presentaba puntuaciones más bajas que el Grupo 1. En Sensibilidad se hallaron diferencias estadísticamente significativas entre el 
Grupo 2 y Grupo 1 siendo las puntuaciones altas para el Grupo 2. En Autosuficiencia las diferencias se encontraron entre los grupos 2 y 1, siendo las puntuaciones más bajas para el Grupo 2. En Perfeccionismo diferencias se encuentran entre los tres grupos, el Grupo 3 presenta puntuaciones más bajas que el grupo 1 y 2 . En Tensión hallamos diferencias estadísticamente significativas entre el Grupo 2 y Grupo 1 siendo las puntuaciones más altas para el Grupo 2. En Infrecuencia encontramos diferencias estadísticamente significativas entre los Grupo 3 y 2 siendo las puntuaciones más altas para el Grupo 3. En Extraversión y Ansiedad las diferencias se encuentran entre los Grupo 2 y 1 siendo las puntuaciones más altas para el Grupo 2 y por último, en Autocontrol encontramos diferencias entre los tres grupos siendo las puntuaciones más bajas en el Grupo 3 y las más altas para el Grupo 2.

\section{Discusión y Conclusiones}

El progreso social y el avance de las Tecnologías de la Información y la Comunicación (TIC) promueven nuevos patrones de comportamientos susceptibles de promover dependencia, como es el abuso del teléfono

Tabla 2

Medias, desviaciones típicas y significación de las variables relacionadas con la personalidad (16 PF-5)

\begin{tabular}{|c|c|c|c|c|c|}
\hline \multirow{2}{*}{$\begin{array}{l}\text { FACTORES } \\
\text { PERSONALIDAD } \\
(16-P F)\end{array}$} & \multicolumn{3}{|c|}{ GRUPOS } & \multirow[b]{2}{*}{$\boldsymbol{F}$} & \multirow[b]{2}{*}{ p-Tukey } \\
\hline & $\begin{array}{c}\text { G1 } \\
\text { (1/2 hora) } \\
\text { Media (DT) }\end{array}$ & $\begin{array}{c}\text { G2 } \\
\text { (<2 horas) } \\
\text { Media (DT) }\end{array}$ & $\begin{array}{c}\text { G3 } \\
\text { (> } 4 \text { horas) } \\
\text { Media (DT) }\end{array}$ & & \\
\hline Afabilidad & $6.06(1.65)$ & $6.53(1.24)$ & $6.30(1.51)$ & 1.455 & \\
\hline Razonamiento & $5.10(1.30)$ & $5.31(1.10)$ & $5.16(1.24)$ & 0.440 & \\
\hline Estabilidad & $4.52(1.21)$ & $4.27(1.45)$ & $3.84(1.38)$ & $3.411^{*}$ & $3<1^{*}$ \\
\hline Dominancia & $4.99(1.93)$ & $5.27(1.85)$ & $4.59(2.02)$ & 1.236 & \\
\hline Animación & $5.92(1.79)$ & $6.71(1.80)$ & 7.24 (1.79) & $7.779 * *$ & $3>1^{\star *} ; 2<1^{*}$ \\
\hline Atención a Normas & $4.23(1.63)$ & $4.22(1.48)$ & $3.51(1.04)$ & 3.362 & \\
\hline Atrevimiento & $4.89(1.42)$ & $5.07(1.99)$ & $5.41(1.30)$ & 1.364 & \\
\hline Sensibilidad & $6.53(1.48)$ & $7.20(1.55)$ & 7.05 (1.65) & $3.268^{*}$ & $2>1^{*}$ \\
\hline Vigilancia & $6.51(1.20)$ & $6.76(1.35)$ & $6.51(1.02)$ & 0.686 & \\
\hline Abstracción & $6.66(1.55)$ & $6.84(1.36)$ & $6.78(1.60)$ & 0.231 & \\
\hline Privacidad & $5.71(1.83)$ & $5.84(1.97)$ & $5.57(1.66)$ & 0.232 & \\
\hline Aprensión & $6.53(1.32)$ & $6.93(1.40)$ & $6.86(1.13)$ & 1.707 & \\
\hline Apertura al Cambio & $6.23(1.97)$ & 5.69 (1.65) & $6.49(1.89)$ & 2.035 & \\
\hline Autosuficiencia & $6.35(1.37)$ & $5.69(1.46)$ & $6.24(1.21)$ & $3.568^{*}$ & $2<1^{*}$ \\
\hline Perfeccionismo & $4.06(1.52)$ & $4.04(1.52)$ & $3.05(1.35)$ & $6.495^{\star *}$ & $3<1^{*} ; 3>2^{* *}$ \\
\hline Tensión & $6.58(1.21)$ & $7.20(1.39)$ & $7.05(1.33)$ & $3.963^{*}$ & $2>1^{*}$ \\
\hline Manipulación de la Imagen & $4.80(1.63)$ & $4.60(1.56)$ & $4.43(1.19)$ & 0.776 & \\
\hline Infrecuencia & $6.84(1.84)$ & $6.22(1.48)$ & $7.62(1.60)$ & $6.921^{* *}$ & $3>2^{* *}$ \\
\hline Aquiescencia & $5.92(1.89)$ & $6.47(1.42)$ & $5.62(1.80)$ & 2.540 & \\
\hline Extraversión & $5.51(1.62)$ & $6.60(1.59)$ & $6.19(1.65)$ & $7.173^{* *}$ & $2>1 * *$ \\
\hline Ansiedad & $6.29(1.86)$ & $7.51(1.95)$ & $6.54(1.92)$ & $6.154^{* *}$ & $2>1^{* *} ; 3>1^{* *}$ \\
\hline Dureza & $5.20(2.12)$ & $5.98(1.80)$ & $5.35(2.15)$ & 2.140 & \\
\hline Independencia & $5.31(1.92)$ & $5.78(1.06)$ & $5.19(1.88)$ & 1.134 & \\
\hline Autocontrol & $4.34(1.93)$ & $4.40(2.07)$ & $2.95(1.35)$ & $8.322^{* * *}$ & $3<1^{* *} ; 2>3^{* *}$ \\
\hline
\end{tabular}

Nota. ${ }^{* * *} p<.001 ;{ }^{* *} p<.01 ;{ }^{*} p<.0$ 
móvil. Conocer y analizar este tipo de comportamientos parece lógico, ya que pueden tener síntomas y consecuencias, al menos en parte, de similares características a las que presentan las personas adictas a alguna sustancia. La población de estudiantes universitarios merece especial atención, pues junto con la adolescencia, son los que presentan mayor sensibilidad a realizar dichas conductas, ya sea por las condiciones en las que viven (fuera del hogar), el escaso control que tienen sobre el dinero, las responsabilidades de la vida diaria, $y / o$ la necesidad que tienen de contactar con los amigos de otros lugares.

El objetivo principal del presente estudio fue analizar las diferencias de personalidad en función del tiempo de uso/abuso del teléfono móvil. Los resultados obtenidos en el presente estudio en las variables del 16PF-5 indican que el Grupo 3 (de abuso del teléfono móvil) caracterizado porque usaba el teléfono móvil más de cuatro horas diarias presentaba puntuaciones bajas en Estabilidad, Atención a las Normas, Perfeccionismo y Autocontrol y puntuaciones altas en Animación y Extraversión. Esto indica que los usuarios que abusan del teléfono móvil son personas afectadas por los sentimientos y que se perturban con facilidad, inconformistas, indulgentes, impulsivos e indiferentes a los fallos y al desorden. Estos resultados se relacionan con los estudios realizados, en esta línea, que indican que el uso del teléfono móvil juega un papel importante en el bienestar subjetivo (Ling, 2005). Aunque, como hemos señalado anteriormente, la investigación no ha sido extensa en la etiología de la adicción a los teléfonos móviles, ya que es un fenómeno nuevo. Los factores psicológicos son quizás, una de las causas más importantes de la adicción al teléfono móvil, si los usuarios piensan que los teléfonos móviles tienen un efecto positivo en sus vidas, tales como mejorar su vida social, pueden ser más propensos a desarrollar una adicción o es bastante probable que abusen del teléfono móvil. En contraste, los usuarios que pueden ver el uso de los teléfonos móviles como una pérdida de tiempo y dinero no desarrollaran una adicción. En este sentido, podemos manifestar que las TIC provocan un híbrido de las funciones cognitivas y estrategias conductuales destinadas a cambiar los comportamientos y las cogniciones, pues existen algunos usuarios que crean una actitud de adaptación y diseñan estrategias de resolución de problemas para comprender el significado de determinadas situaciones y emociones. El teléfono móvil es utilizado como un cordón umbilical simbólico para conectar los adolescentes con sus padres y además, se ha convertido en una herramienta de la emancipación, esto es, da al adolescente una fuente de interacción que no está mediada por los padres y que desempeña la cohesión social del grupo de pares. Destacar también, que estos resultados son congruentes con el estudio de Ishfaq et al. (2011) que encontró que los jóvenes, en muchos casos, utilizan el teléfono móvil como estrategias de afrontamiento de estados emocionales negativos tales como aburrimiento o disforia. Estos resultados son congruentes con los obtenidos por Choliz (2012) que encuentran una relación directa y estadísticamente significativa entre el grado de dependencia y el malestar emocional cuando no pueden usar el teléfono móvil, así como otros factores como el uso excesivo, problemas con los padres, la dificultad en el control de la utilización y la interferencia con otras actividades.

Con respecto al Grupo 2 (uso del teléfono móvil) encontramos puntuaciones altas en Extraversión y Ansiedad estos resultados son congruentes con otros estudios (Bianchi y Philips, 2005; Ishfag et al., 2011; Turner et al., 2008) que afirman que el teléfono móvil puede generar modificaciones del estado de ánimo, ansiedad, sentimiento de inseguridad, miedo a salir él, tener que volver a por él si se olvida y/o no ser capaz de hacer nada sin el teléfono móvil.

Por lo tanto, los extrovertidos pueden utilizar sus teléfonos con más frecuencia, pero no necesariamente en situaciones donde pueden ser supervisados por los demás. Si se supone que fisiológica intrínseca la excitación será mayor al realizar conversaciones telefónicas en la presencia física de otros, estos hallazgos parecen consistentes con la explicación de que los extrovertidos son más dependientes de la telefonía móvil para el desarrollo social debido a su probabilidad de tener mayor redes sociales e inconsistente con la opinión de que los extrovertidos utilizan los teléfonos 
móviles para crear una mayor activación fisiológica (Bianchi y Phillips, 2005). En este sentido, de acuerdo con los estudios de Hong et al. (2012) se pone de manifiesto que la extraversión se relaciona con una mayor frecuencia de uso del teléfono móvil, puesto que la extraversión predice, de manera positiva, la adicción al teléfono móvil.

Los resultados del presente estudio ponen de manifiesto la necesidad de seguir investigando, con el fin de, identificar a los usuarios que están en riesgo de adicción del teléfono móvil, establecer las variables que predisponen el desarrollo de patología del uso del teléfono móvil y desarrollar estrategias de intervención, prevención e indicaciones para los educadores para reducir el uso adictivo de los teléfonos móviles en los adolescentes y jóvenes universitarios. La investigación futura debería ampliar el alcance de este estudio, comparando los resultados de diferentes grupos de historia de uso del tiempo libre y examinar otras variables dependientes, como el rendimiento académico y las redes sociales como resultado del uso adictivo de la telefonía móvil. Los estudios futuros deberían centrarse, además, en los patrones de adaptación frente a los desadaptativos en el uso del teléfono móvil y ofrecer algunas indicaciones para el uso responsable del móvil en relación con la intervención y con el diseño de estrategias encaminadas a reducir el uso adictivo de los teléfonos móviles de la población. Entre las limitaciones de este estudio encontramos que la recogida de datos se ha llevado a cabo con población universitaria; sin embargo, a pesar de esta situación consideramos que los datos obtenidos son válidos y extrapolables, ya que los universitarios representan una parte bastante amplia de la sociedad juvenil en nuestro país, y son en consecuencia, un alto porcentaje de usuarios de teléfonos móviles. Destacamos también que en nuestra muestra de estudio las mujeres están mayoritariamente representadas, sin embargo esta realidad es consonante con los estudios realizados por Hong et al. (2012) con población femenina y por Jenaro et al. (2007) quienes hallaron que las mujeres abusan más del teléfono móvil que los hombres y finalmente, otra limitación destacada que deriva de este estudio es el impresionante crecimiento de las funciones y aplicaciones tecnológicas de los teléfonos móviles. El uso de Internet por el móvil, o el desarrollo de aplicaciones como WhatsApp, no sólo modifica el patrón de uso de los teléfonos móviles, pero son algunas de las variables cruciales que también pueden inducir el abuso o la dependencia del teléfono móvil. Esto es particularmente relevante para los adolescentes, debido a la dependencia de los adolescentes en el teléfono móvil es un problema que no sólo es nueva, sino que también va en aumento. Por tanto, es necesario seguir investigando las condiciones que promuevan esta dependencia, para desarrollar programas de prevención y tratamiento, y para hacer la evaluación disponible y los instrumentos de diagnóstico que permiten una intervención eficaz.

\section{Referencias}

Aluja, A. y Blanch, A. (2003). Replicabilidad de los factores de segundo orden del 16PF-5 en muestras americanas y españolas. Psicothema, 15(2), 309314.

American Psychiatric Association (2011). Diagnostic and Statistical Manual of Mental Disorders ( $4^{\mathrm{a}}$ ed.). Washington, DC: Autor.

Beck, A. T. y Steer, R. (1993a). Beck Anxiety Inventory manual. San Antonio, TX: Psychological Corporation.

Beck, A. T. y Steer, R.A. (1993b). Beck Depression Inventory manual. San Antonio, TX: The Psychological Corporation.

Bianchi, A. y Phillips, J. G. (2005). Psychological predictors of problem mobile phone use. Cyberpsychology \& Behavior, 8, 39-51. http://dx.doi.org/10.1089/cpb.2005.8.39

Bononato, L. B. (2005). Adicciones y nuevas tecnologías. Proyecto Hombre, Ciberadicciones, 55, 17-21.

Cattell, R. B., Cattell, A. K. S. y Cattell, H. E. P. (2005). Cuestionario Factorial de Personalidad ( $8^{\mathrm{a}} \mathrm{Ed}$. Rev.). Madrid, España: TEA. 
Chóliz, M. (2012). Mobile-phone addiction in adolescence: The Test of Mobile Phone Dependence (TMD). Progress in Health Sciences, 2, 33-44.

Echeburúa, E., Salaberría, K. y Cruz-Sáez, M. (2014). Nuevos Retos en el Tratamiento del Juego Patológico. Terapia Psicológica, 32, 31-40.

Eysenck, H. J. y Eysenck, S. B. G. (1991). Manual of the Eysenck Personality Scales (EPS Adult). Londres, UK: Hodder \& Stoughton.

Francis, L. J., Brown, L. B. y Philipchalk, R. (1992). The development of an abbreviated form of the revised Eysenck personality questionnaire (EPQR-A): its use among students in England, Canada, the USA and Australia. Personality and Individual Differences, 13, 443-449. http://dx.doi.org/10.1016/0191-8869(92)90073-X

Hong, F., Chiu, S. y Huang, D. (2012). A model of the relationship between psychological characteristics, mobile phone addiction and use of mobile phones by Taiwanese university female students. Computers in Human Behavior, 28, 2152-2159. http://dx.doi.org/10.1016/j.chb.2012.06.020

Howell, M., Love, S. y Turner, M. (2005). The impact of spatial metaphor and context of use on the usability of a speech-based mobile city guide service. Behaviour \& Information Technology, 24, 67-78.

Ishfaq, A., Tehmina, F. Q. y Khadija, A. P. (2011). Mobile Phone to Youngsters: Necessity or Addiction. Information Management and Business Review, 2, 229-238. http://dx.doi.org/10.5897/AJBM11.626

Jenaro, C., Flores, N., Gómez-Vela, M., González-Gil, F. y Caballo, C. (2007). Problematic internet and cellphone use: Psychological, behavioral, and health correlates. Addiction Research and Theory, 15, 309320. http://dx.doi.org/10.1080/16066350701350247
Labrador, F. J. y Villadangos, S. M. (2010). Menores y nuevas tecnologías: Conductas indicadoras de posible problema de adicción. Psicothema, 22, 180188.

Lai, B. Z. y Lai, M. L. (2003). New Lai's Personality Scale Guide Handbook. Taipei, China: Chian Hua.

Leung, L. (2007). Unwillingness-to-communicate and College Students' Motives in SMS Mobile Messaging. Telematics \& Informatics, 24, 115-129. http://dx.doi.org/10.1016/j.tele.2006.01.002

Ling, R. (2005). Mobile communications vis-à-vis teen emancipation, peer group integration and deviance. En R. Harper, A. Taylor y L. Palen (Eds.), Social perspectives on SMS in the mobile age (pp. 175189). Londres, UK: Klewer.

Lobo, A., Pérez-Echeverría, M. J. y Artal, J. (1986). Validity of the scaled version of the General Health Questionnaire (GHQ-28) in a Spanish population. Psychological Medicine, 16, 135-140. http://dx.doi.org/10.1017/S0033291700002579

Muñoz-Rivas, M. J. y Agustín, S. (2005). La adicción al teléfono móvil. Psicología Conductual, 13, 481-493.

Rosenberg, M. (1965). Society and the adolescent selfimage. Princeton, NJ: Princeton University Press.

Ruiz-Olivares, R., Lucena, V., Pino, M. y Herruzo, J. (2010). Análisis de comportamientos relacionados con el uso/abuso de Internet, teléfono móvil, compras y juego en estudiantes universitarios. Adicciones, 22, 301-310.

Sánchez-Carbonell, X., Beranuy, M., Castellana, M., Chamarro, A. y Oberst, U. (2008). La adicción a Internet y al móvil: ¿moda o trastorno? Adicciones, 20, 149-160.

Srivastava, L. (2005). Mobile phones and the evolution of social behaviour. Behaviour \& Information Technology, 24, 111-129. http://dx.doi.org/10.1080/01449290512331321910 
Turner, M., Love, S. y Howell, M. (2008). Understanding emotions experienced when using a mobile phone in public: The social usability of mobile (cellular) telephones. Telematics and Informatics, 25, 201-215.

Viñas, F. y González, M. (2010). Amenazas a través de la telefonía móvil e internet: perfil psicológico y consecuencias emocionales [Threats through mobile phones and internet: psychological profile and emotional consequences]. Acción Psicológica, 7(1), 31-40. http://dx.doi.org/10.5944/ap.7.1.205

Walsh, S. P., White, K. M., Cox, S. y Young, R. M. (2011). Keeping in constant touch: The predictors of young Australians' mobile phone involvement. Computers in Human Behavior, 27, 333-342. http://dx.doi.org/10.1016/j.chb.2010.08.011

Watson, D., Clark, L. A. y Tellegen, A. (1988). Development and validation of brief measures of positive and negative affect: The PANAS scales. Journal of Personality and Social Psychology, 54, 1062-1070. http://dx.doi.org/10.1037/00223514.54.6.1063

Weed, N. C., Butcher, J. N. y McKenna, T. (1992). New measures for assessing alcohol and drug abuse with the MMPI-2: the APS and the AAS. Journal of Personality Assessment, 58, 389-404. http://dx.doi.org/10.1207/s15327752jpa5802_15

Young, K. S. (1996). Psychology of computer use: XL. Addictive use of the internet: a case study that breaks the stereotype. Psychological Reports, 79, 899-902.

http://dx.doi.org/10.2466/pr0.1996.79.3.899

Young, K. S. (1998). Internet addiction: The emergence of a new clinical disorder. Cyberpsychology and Behavior, 1 , 237-244. http://dx.doi.org/10.1089/cpb.1998.1.237 